\title{
Analysis of Risk Factors for Lymphatic Metastasis in Endometrial Carcinoma and Utility of Three-Dimensional Magnetic Resonance Imaging in Gynecology
}

\author{
Yasuhito Tanase $^{\mathrm{a}, \mathrm{c}}$, Junko Takahama ${ }^{\mathrm{b}}$, Ryuji Kawaguchi ${ }^{\mathrm{a}}$, Hiroshi Kobayashi ${ }^{\mathrm{a}}$
}

\begin{abstract}
Background: The aim of our study was to evaluate the utility of three-dimensional magnetic resonance imaging (3D-MRI) in gynecologic fields. We examined the relation between tumor volume measured with 3D-MRI and lymph node metastasis in patients with endometrial carcinoma.
\end{abstract}

Methods: A retrospective analysis of 84 patients with endometrial carcinoma who underwent hysterectomy, bilateral salpingo-oophorectomy with pelvic/para-aortic lymphadenectomy at our institute was performed. Of these, the tumor volume of 59 patients could be calculated using 3D-MRI. Age, serum CA125 level, histologic type and grade, volume of tumors were examined in relation to pelvic/ para-aortic lymph node metastasis as preoperative risk factors. Tumor volume measurements were calculated using 3D-MRI with AqariusNET Server 4G software. Univariate and multivariate associations between the preoperative risk factors and pelvic/para-aortic lymph node metastasis were analyzed. Receiver operating characteristic (ROC) curves were used to determine the best cut-off points for CA125 levels and tumor volume to predict lymph metastasis.

Results: The mean age, CA125 value and tumor volume were 61.6 years, $51.6(\mathrm{IU} / \mathrm{L})$ and $11.6\left(\mathrm{~cm}^{3}\right)$, respectively. Lymphatic metastasis occurred in $16.0 \%$ (10 of 59) patients. Univariate analysis indicated that a high CA125 level and a tumor volume were risk factors ( $\mathrm{P}=$ $0.0111,0.0123$ respectively). Multivariate analysis revealed that tumor volume was an independent risk factor for lymphatic metastasis (hazard ratio (HR) $12.7,95 \%$ CI 1.06 - 154). The potential cut-off values of CA-125 level and tumor volume were 29 IU/L (sensitivity: 0.744 ; specificity: 0.821 ) and $12.79 \mathrm{~cm}^{3}$ (sensitivity: 0.821 ; specificity: 0.744$)$, respectively.

Conclusions: Our results suggest that tumor volume calculated with 3D-MRI correlates with lymph node metastasis in endometrial car-

Manuscript submitted April 15, 2018, accepted April 28, 2018

${ }^{a}$ Department of Obstetrics and Gynecology, Nara Medical University, Kashihara, Nara, Japan

bDepartment of Radiology, Nara Medical University, Kashihara, Nara, Japan ${ }^{\mathrm{c} C o r r e s p o n d i n g ~ A u t h o r: ~ Y a s u h i t o ~ T a n a s e, ~ S h i j o-c h o ~ 840, ~ K a s h i h a r a, ~ N a r a, ~}$ 634-8522, Japan.Email: ytana517@naramed-u.ac.jp

doi: https://doi.org/10.14740/wjon1106w cinoma.

Keywords: Endometrial neoplasms; Three-dimensional magnetic resonance imaging; Lymphatic metastasis; Tumor volume

\section{Introduction}

Some well-known risk factors for retroperitoneal lymph node metastasis in endometrial cancer include histologic grade, myometrial invasion, volume index, and serum CA125 levels. Of these, tumor volume has been reported to be one of the risk factors of pelvic/para-aortic lymph node metastasis [1]. Conventionally, tumor volume has been estimated from crosssectional area, which is calculated as the product of the longest diameter and longest perpendicular diameter. However, in the present study we calculated tumor volume in endometrial carcinoma using three-dimensional magnetic resonance imaging (3D-MRI) with AqariasNet Surver 4G software.

Although there have been many reports regarding the utility of 3D-compted tomography (CT) in gynecologic fields [2], there are very few that investigate the utility of 3D-MRI. Associated with lymphatic metastasis and 3D-CT the utility has been reported in other fields $[3,4]$. MRI has been reported to have many advantages in pelvic evaluation [5-8]. We expect that by using 3D-MRI reconstructions more complete visualization and evaluation of the pelvis were possible, and examined the usefulness of 3D-MRI in gynecological fields in this article. We hypothesize that 3D-MRI is equivalent or more compared to 3D-CT in pelvic reconstructions. The aim of our study was to evaluate the utility of 3D-MRI in gynecologic fields. We examined the relation between tumor volume measured with 3D-MRI and retroperitoneal lymph node metastasis in patients with endometrial carcinoma.

\section{Materials and Methods}

\section{Patients}

The study protocol was approved by the Institutional Review Board of the university, and written informed consent was tak- 


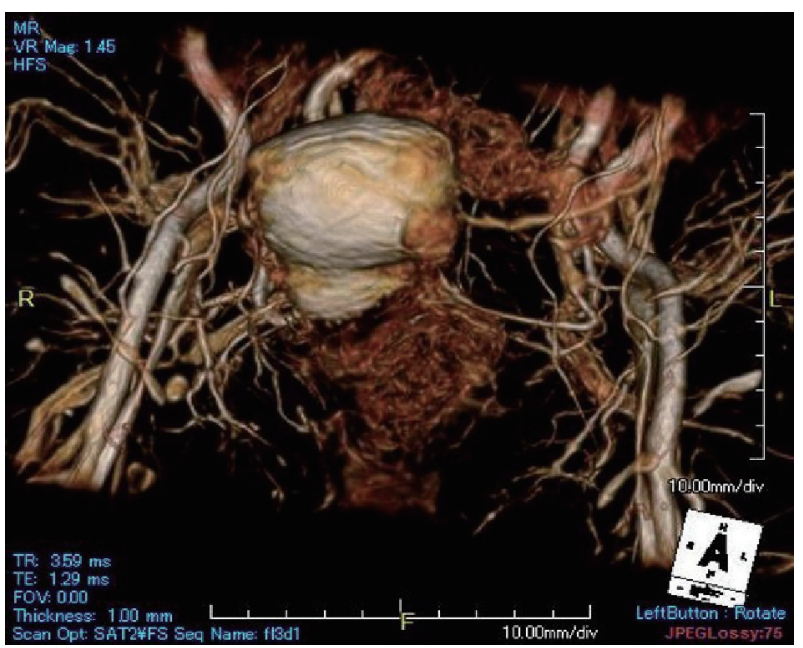

Figure 1. Using 3D-MRI reconstructions complete visualization of the pelvis is possible.

en from all patients. Between April 2011 and April 2016, 84 patients with endometrial adenocarcinoma underwent hysterectomy and bilateral salpingo-oophorectomy with pelvic/paraaortic lymphadenectomy in the Department of Obstetrics and Gynecology, Nara Medical University. Of those 84 patients with endometrial cancer, we selected 59 patients whose preoperative MRI had been performed in our institution and could be applied to the software, AqariusNET Server 4G. We excluded 25 patients who did not apply to the above. Of these, the tumor volume of 59 patients could be calculated using 3D-MRI.Pelvic lymphadenectomy had been performed in 33 patients, and pelvic + paraaortic lymphadenectomy in 26 patients.

\section{Subjects}

Age, serum CA125 level, preoperative histologic type and grade, and MRI finding of depth of uterine wall of tumors were examined in relation to pelvic/para-aortic lymph node metastasis as preoperative risk factors. In addition, the tumor volume of 59 patients could be calculated using 3D-MRI. Associated with preoperative histologic type and grade, endometrioid carcinoma grade1, grade 2, grade 3 were indicated G1, G2, G3 respectively, and non-endometrioid carcinoma including serous carcinoma, clear cell carcinoma was indicated others. Associated with depth of the uterine wall of tumors, less than one half or more of the myometrial invasion diagnosed by MRI were examined. Added to those, postoperative diagnosis of histologic type and depth of the uterine wall were examined with pathological specimen. The frequencies of upgrade and upstaging were also analyzed. All pathological sections were analyzed by a highly qualified pathologist who was a board certified by the Japanese Society of Pathology.

\section{Image analysis and tumor volume setting}

Tumor volume measurements were calculated using 3D-MRI

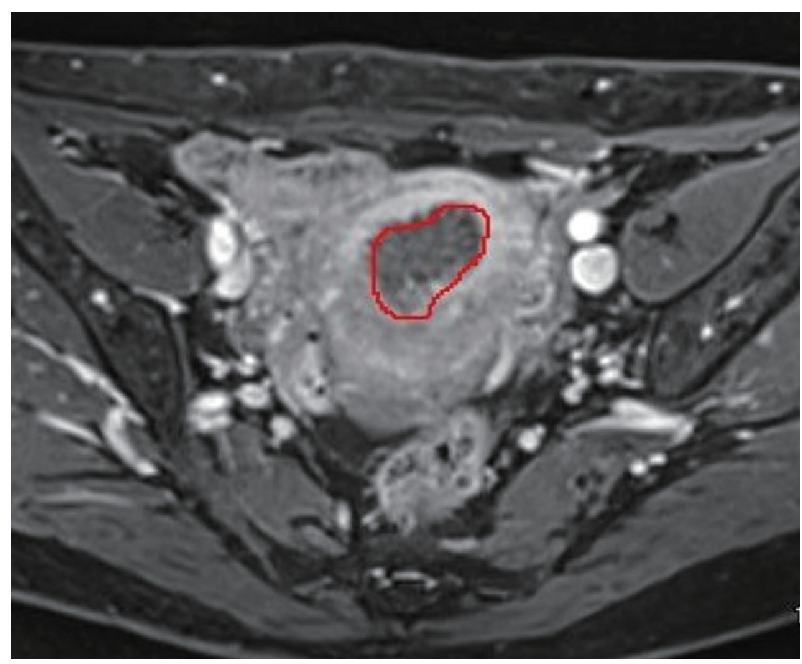

Figure 2. The border of tumors is outlined and the outlined tumors are cored out, integrated, and the tumor volume is automatically calculated using the volumetry function.

with AqariusNET Server 4G software. Acquired MRI images were transferred to the software, which allowed for 3D views (Fig. 1) and volumetry. The tumor margins were determined from T2-weghted images or T1-weighted images enhanced by gadolinium, which showed tumor lesions more clearly. The first step for calculating the tumor volume is outlining the border of the tumors; we put region of interest (ROI) at the tumor manually. Using the volumetry function within the software, the outlined tumors are cored out, integrated, and the tumor volume is automatically calculated (Fig. 2). MRI finding of tumors were often varied, and there were some cases in which the tumors had necrosis or unclear boarder. In those cases we took the parts of tumors which were enhanced by gadolinium clearly. Under the direction of one expert radiologist with more than 20 years' of experience, MRI findings were examined by one gynecologic oncologist with more than 10 years' of experience. They were not involved in the clinical care. We performed ROI measurement twice with each case and took average of them. MR imaging was obtained on a 3Tesra system (Magnetom Verio, Siemens Healthcare, Erlangen, Germany) with 32-element body array coil. The protocol of our routine MR examination was as follows; T2WI (sagittal) (TSE: TR/ $\mathrm{TE}=4000 / 89$, Matrix $=512 / 307, \mathrm{FOV}=200 \times 200, \mathrm{ETL}=19$, slice thickness $=3 \mathrm{~mm}), \mathrm{T} 1 \mathrm{WI}($ axial) $(\mathrm{SE}: \mathrm{TR} / \mathrm{TE}=450 / 12$, Matrix $=320 / 192$, FOV $=200 \times 200$, slice thickness $=3 \mathrm{~mm})$, T2WI (axial) (SPACE: TR/TE $=2700 / 287$, Matrix $=256 / 256$, $\mathrm{FOV}=250 \times 250$, slice thickness $=1.0 \mathrm{~mm}$, flip angle mode $=\mathrm{T} 2$ var), HASTE (coronal) $\mathrm{TR} / \mathrm{TE}=2000 / 88$, Matrix $=$ $320 / 240, \mathrm{FOV}=280 \times 280$, slice thickness $=5 \mathrm{~mm})$. All cases underwent dynamic contrast-enhanced MRI with a dose of $10 \mathrm{~mL}$ of meglumine gadopentetate (Magnevist®; Bayer, Germany), that was injected with an automated injector at a rate of $2 \mathrm{~mL} / \mathrm{s}$ and followed by a $20 \mathrm{~mL}$ saline flush. Dynamic MRI was performed at three continuous fat saturated T1 weighted images (axial) (VIBE: TR/TE $=3.74 / 1.38, \mathrm{FA}=11$, Matrix $=$ $256 / 256, \mathrm{FOV}=250 \times 200$, slice thickness $=1.0 \mathrm{~mm})$ in 60 , $120,180 \mathrm{~s}$ after injection. These diagnostic imaging modalities 
Table 1. Clinicopathologic Features of 59 Patients With Endometrial Adenocarcinoma

\begin{tabular}{|c|c|c|c|}
\hline Factor & & No. & $\%$ \\
\hline \multicolumn{4}{|l|}{ Age (years) } \\
\hline Mean \pm SD & $61.6 \pm 7.7$ & & \\
\hline$\geq 60$ & & 34 & 57.6 \\
\hline$<60$ & & 25 & 42.4 \\
\hline \multicolumn{4}{|c|}{ Histologic type (preoperative) } \\
\hline G1 & & 28 & 47.5 \\
\hline G2 & & 11 & 18.6 \\
\hline G3 & & 5 & 8.5 \\
\hline Others & & 15 & 25.4 \\
\hline \multicolumn{4}{|c|}{ Myometrial invasion (MRI) } \\
\hline$\geq 1 / 2$ & & 25 & 42.4 \\
\hline$<1 / 2$ & & 34 & 57.6 \\
\hline \multicolumn{4}{|l|}{ CA125 (IU/L) } \\
\hline Mean \pm SD & $51.6 \pm 135.3$ & & \\
\hline$\geq 29$ & & 20 & 33.2 \\
\hline$<29$ & & 39 & 66.1 \\
\hline \multicolumn{4}{|c|}{ Tumor volume $\left(\mathrm{cm}^{3}\right)$} \\
\hline Mean \pm SD & $11.6 \pm 18.4$ & & \\
\hline$\geq 12.79$ & & 27 & 45.8 \\
\hline$<12.79$ & & 32 & 54.2 \\
\hline \multicolumn{4}{|c|}{ Lymphatic metastasis } \\
\hline positive & & 10 & 16.9 \\
\hline negative & & 49 & 83.1 \\
\hline
\end{tabular}

are acceptable for excluding the possibility of malignancy before surgery and choosing appropriate treatment option.

\section{Statistical analysis}

Logistic regression analysis was used to evaluate univariate and independent multivariate associations between the various preoperative risk factors and pelvic/para-aortic lymph node metastasis. Receiver operating characteristic (ROC) curves were used to determine the best cut-off points for serum CA125 levels and tumor volume to predict lymph metastasis. Analyses

Table 2. Evaluation of Histologic Grade

\begin{tabular}{llllll}
\hline & & \multicolumn{5}{c}{$\begin{array}{c}\text { Postoperative } \\
\text { histologic grade }\end{array}$} \\
\cline { 3 - 7 } & & G1 & G2 & G3 & Others \\
\hline \multirow{3}{*}{ Preoperative histologic grade } & G1 & 22 & 4 & 0 & 2 \\
& G2 & 1 & 9 & 1 & 0 \\
& G3 & 0 & 0 & 5 & 0 \\
& Others & 1 & 1 & 2 & 11 \\
\hline
\end{tabular}

Table 3. Evaluation of Myometrial Invasion

\begin{tabular}{llll}
\hline & & \multicolumn{2}{c}{$\begin{array}{c}\text { Myometrial invasion } \\
\text { by pathology }\end{array}$} \\
\hline \multirow{2}{*}{ MRI based myometrial invasion } & $<1 / 2$ & $\geq 1 / 2$ \\
& $<1 / 2$ & 29 & 5 \\
& $\geq 1 / 2$ & 2 & 23 \\
\hline
\end{tabular}

were performed using commercially available software packages (SPSS for Windows version 17.0 and Medcalc for Windows version 11.4.2.0). Values of $\mathrm{P}<0.05$ were deemed to indicate statistical significance.

\section{Results}

Table 1 shows the clinicopathologic characteristics of 59 patients with endometrial cancer and the preoperative histologic type. The mean age was 61.6 years. Preoperatively, 66\% (39 of 59 ) were diagnosed as G1 and G2 cases and 5\% (three of 59) of these were upgraded to G3 adenocarcinoma or others after surgery (Table 2). Cases of less than one half the myometrial invasion diagnosed by MRI were $57.6 \%$ (34 of 59). The 3DMRI showed a sensitivity of $82.1 \%$ for preoperative diagnosis, a specificity of $93.5 \%$, and a diagnostic accuracy of $88.1 \%$ associated with myometrial invasion of the tumors (Table 3 ). The mean CA125 value and tumor volume were 51.6 (IU/L) and $11.6\left(\mathrm{~cm}^{3}\right)$, respectively. Lymphatic metastasis occurred in $16.0 \%$ (10 of 59) patients. Of those 10 cases, pelvic lymphatic metastasis was observed in four cases, paraaortic lymphatic metastasis was in three cases and both of them were in three cases.

ROC analysis was used to determine potential cut-off values of CA-125 level and tumor volume for prediction of lymphatic metastasis (Fig. 3, 4). For CA125 level the cut-off value was $29 \mathrm{IU} / \mathrm{L}$ (area under the curve (AUC) 0.7062, 95\% confidence interval (CI): 0.5224 - 0.8901), which demonstrated a sensitivity and specificity of 0.744 and 0.821 , respectively. The cut-off value of the tumor volume was $12.79 \mathrm{~cm}^{3}$. (AUC 0.7933, 95\% CI: 0.6685 - 0.9193), which demonstrated a sensitivity and specificity of 0.821 and 0.744 , respectively.

Table 4 shows the regression analysis between the preoperative factors and lymphatic metastasis in endometrial adenocarcinoma. Univariate analysis indicated that a high serum CA125 level $(\geq 29)$ and a tumor volume of $\geq 12.79$ were risk factors $(\mathrm{P}$ value $=0.0111,0.0123$ respectively $)$. Furthermore, multivariate analysis revealed that tumor volume of $\geq 12.79$ was an independent risk factor for lymphatic metastasis (HR $12.7,95 \%$ CI 1.06 - 154, P value $=0.04)$.

\section{Discussion}

We examined the preoperative risk factors for pelvic/para-aortic lymph node metastasis in endometrial carcinoma. Our results suggest that serum CA125 levels and tumor volume calculated using 3D-MRI correlated with retroperitoneal lymph 


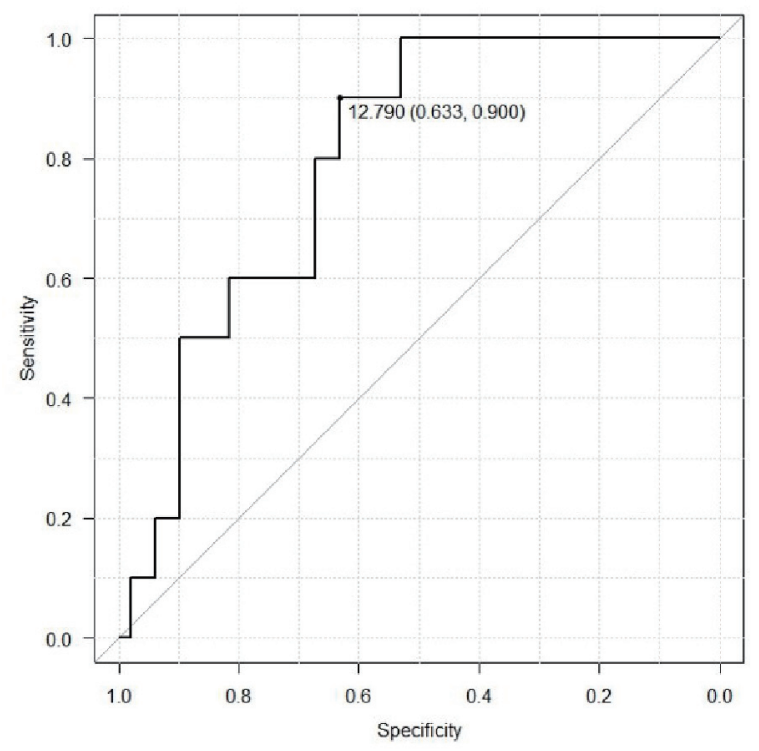

Figure 3. Receiver operating curve to determine potential values of tumor volume for prediction of lymphatic metastasis.

node metastasis. Tumor volume, in particular, was strongly associated with lymph node metastasis (Table 4). It has been reported that elevations in serum CA125 level are strongly associated with advanced surgical stage, lymph node metastasis, and poor prognosis $[8,9]$. In the present study, only univariate analyses indicated that CA125 level $(<29,(\geq 29))$ was a risk factor. Yidiz et al reported that high CA125 levels significantly correlated with advanced stage and lymph node metastases in endometrial carcinoma [10]. In their study, the ROC curve determined that the best cut-off value was $20 \mathrm{U} / \mathrm{mL}$ with a sensitivity and specificity of $75 \%$ and $69.51 \%$, respectively, and a positive predictive value and negative predictive value of $80.6 \%$ and $84.9 \%$, respectively. While they concluded that CA125 appeared to be a significant independent predictor of the advanced stage of the disease as well as lymph node metastases, more studies are needed to establish the appropriate cut-off level for serum CA125.

Todo et al reported the utility of the tumor volume index to stratify the risk for lymph node metastasis in endometrial carcinoma [1]. They concluded that the tumor volume index in endometrial carcinoma was an independent risk factor for

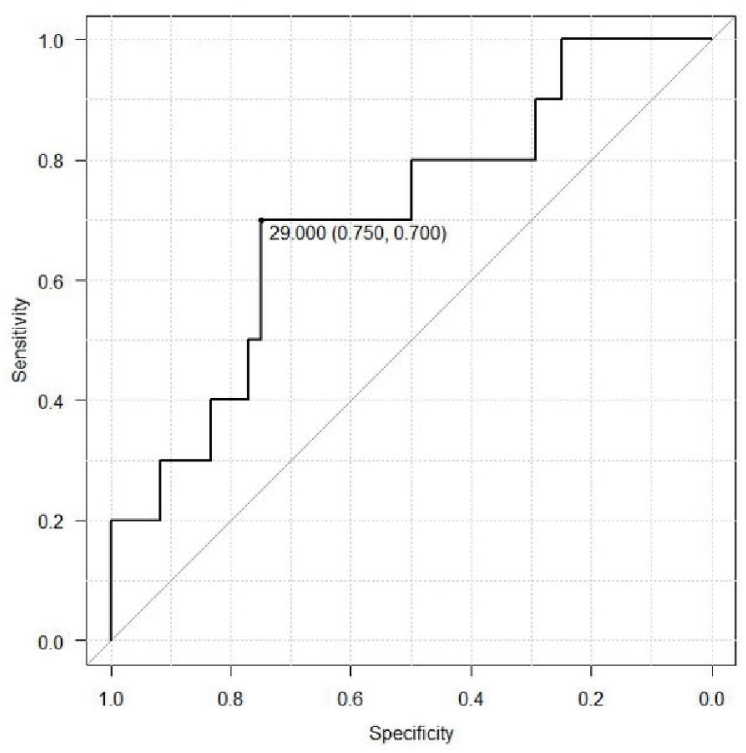

Figure 4. Receiver operating curve to determine potential values of CA125 level for prediction of lymphatic metastasis.

pelvic/para-aortic lymph node metastasis. In their study they defined the volume index as the product of maximum longitudinal, anteroposterior, and horizontal diameter. In contrast to their study we calculated tumor volume by integral using the software, AqueriasNet Surver 4G. Our novel method is new and superior for calculating tumor volume, theoretically. In endometrial carcinoma as well as other types of tumors, volume has been related to lymph metastasis and prognosis. Joo $\mathrm{YH}$ et al reported that, in oral tongue cancer, tumor volume larger than $20 \mathrm{~cm}^{3}$ was associated with a greater risk of cervical lymph node metastasis and poor prognosis [11]. Fu et al reported positive correlations between tumor volume and the presence of nodal metastasis [12]. They concluded that tumor volume $(>10 \mathrm{~mL})$ in nasopharyngeal carcinoma is a potential indication for neck irradiation.

The effectiveness of 3D-MRI has been reported, particularly for MRI supported by 3D-MRI reconstructions for complete visualization of the pelvis [13-16]. Giusti et al investigated anatomical localization of deep infiltrating endometriosis. They concluded that the addition of 3D-MRI reconstructions improved diagnostic accuracy and staging of endometriosis

Table 4. Correlation Between the Preoperative Risk Factors and Lymphatic Metastasis

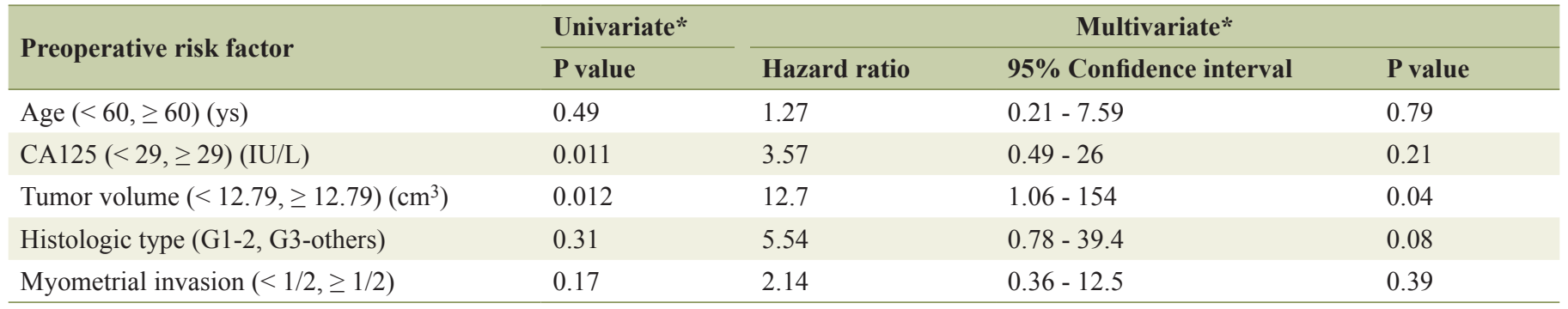

*Logistic regression analysis. 
[17]. Chen et al measured breast density using 3D-MRI and correlated it with endogenous hormone levels during menstrual cycle [18]. They used a semiautomatic computer-based algorithm for the segmentation of the breast region and the fibroglandular tissue and calculated breast density. We used a similar algorithm in the present study. 3D-MRI is likely to be very useful and applicable to many gynecologic fields.

Although it would be important to demonstrate a correlation between lymphatic metastasis and tumor volumes for the purpose of predicting a preoperative risk factor, in the present study we did not compare tumor volumes with surgical specimens. In fact, there are few studies that compare tumor volumes from different imaging modalities with those of surgical specimens. However, to calculate the volume of surgical specimens, the whole-mount serial section technique has been reported, including breast cancer, lung cancer, and laryngeal cancer [1921]. Zhang et al compared cervical cancer tumor volume using MRI, CT, and fluorine-18 fluorodeoxyglucose position emission tomography $\left({ }^{18} \mathrm{~F}-\mathrm{FDG} \mathrm{PET}\right)$ with that of surgical specimens [22]. They concluded that PET-based tumor volume was the most accurate method of the three imaging modalities, but MRI provided a better visualization of larger tumors.

It appears that $3 \mathrm{D}$ volumetric methods should be more accurate than $2 \mathrm{D}$ methods for determining tumor size. The main advantage of using a 3D volumetric method is that it allows more accurate measurements of irregular tumors. Mei-Yun Wang et al reported the usefulness of 3D-MRI in calculating tumor volumes of adult glioblastoma, particularly because cystic components can easily be excluded from the volume analysis [23].

\section{Limitations}

There are several limitations to the present study. First, the subject number is small, which may not be sufficient to observe a strong association. Second, because subjects were limited, retroperitoneal lymphatic metastasis included both pelvic and para aortic lymph nodes, which would normally be dealt with separately. Third, volume measurement using 3D-MRI is very time consuming to perform, which makes it difficult as a routine method; and other types of evaluation of tumor volume are needed to assess the accuracy of 3D-MRI. Fourth, it was a retrospective study, and there may have some selection bias. Further, preferably prospective studies are needed. It would be necessary to increase cases, set a more accurate cut-off level of tumor volume, and examine the values prospectively.

\section{Conclusions}

In conclusion, preoperative CA125 level in combination with the measurement of tumor volume using 3D-MRI were significant for predicting lymphatic metastasis. When used together, these predictive may allow clinicians to stratify patients that need lymphadenectomy in the early stages of uterine endometrioid adenocarcinoma. Although the optimal cut-off levels vary to some extent, elevated CA125 levels and large tumor volumes in patients with apparent early-stage disease place them in a higher risk category for the presence of extra-uterine disease.

\section{Conflict of Interest}

The authors declared no conflict of interest.

\section{Author Contributions}

Yasuhito Tanase: data collection and writing; Junko Takahama: analysis of MRI; Ryuji Kawaguchi: data analysis; Hiroshi Kobayashi: original proposer and proof.

\section{References}

1. Todo Y, Sakuragi N, Nishida R, Yamada T, Ebina Y, Yamamoto R, Fujimoto S. Combined use of magnetic resonance imaging, CA 125 assay, histologic type, and histologic grade in the prediction of lymph node metastasis in endometrial carcinoma. Am J Obstet Gynecol. 2003;188(5):1265-1272.

2. Nakamura M, Fujii T, Imanishi N, Jinzaki M, Yamada M, Kuribayashi S, Aoki D. Surgical anatomy imaging associated with cervical cancer treatment: A cadaveric study. Clin Anat. 2014;27(3):503-510.

3. Giesel FL, Fiedler H, Stefanova M, Sterzing F, Rius M, Kopka K, Moltz JH, et al. PSMA PET/CT with Gluurea-Lys-(Ahx)-[(6)(8)Ga(HBED-CC)] versus 3D CT volumetric lymph node assessment in recurrent prostate cancer. Eur J Nucl Med Mol Imaging. 2015;42(12):17941800.

4. Nagata K, Endo S, Kudo SE. [New preoperative imaging and navigation systems for the treatment of colorectal cancer]. Nihon Geka Gakkai Zasshi. 2008;109(2):95-100.

5. Zamani N, Modares Gilani M, Zamani F, Zamani MH. Utility of pelvic MRI and tumor markers HE4 and CA125 to predict depth of myometrial invasion and cervical involvement in endometrial cancer. J Family Reprod Health. 2015;9(4):177-183.

6. Kuang F, Yan Z, Li H, Feng H. Diagnostic accuracy of diffusion-weighted MRI for differentiation of cervical cancer and benign cervical lesions at 3.0T: Comparison with routine MRI and dynamic contrast-enhanced MRI. J Magn Reson Imaging. 2015;42(4):1094-1099.

7. Fukunaga T, Fujii S, Inoue C, Kato A, Chikumi J, Kaminou $\mathrm{T}$, Ogawa $\mathrm{T}$. Accuracy of semiquantitative dynamic contrast-enhanced MRI for differentiating type II from type I endometrial carcinoma. J Magn Reson Imaging. 2015;41(6):1662-1668.

8. Takeshima N, Shimizu Y, Umezawa S, Hirai Y, Chen JT, Fujimoto I, Yamauchi K, et al. Combined assay of serum levels of CA125 and CA19-9 in endometrial carcinoma. Gynecol Oncol. 1994;54(3):321-326.

9. Sood AK, Buller RE, Burger RA, Dawson JD, Sorosky JI, Berman M. Value of preoperative CA 125 level in the 
management of uterine cancer and prediction of clinical outcome. Obstet Gynecol. 1997;90(3):441-447.

10. Yildiz A, Yetimalar H, Kasap B, Aydin C, Tatar S, Soylu F, Yildiz FS. Preoperative serum CA 125 level in the prediction of the stage of disease in endometrial carcinoma. Eur J Obstet Gynecol Reprod Biol. 2012;164(2):191-195.

11. Joo YH, Hwang SH, Sun DI, Cho KJ, Park JO, Kim MS. Relationships between tumor volume and lymphatic metastasis and prognosis in early oral tongue cancer. Clin Exp Otorhinolaryngol. 2013;6(4):243-248.

12. Fu J, Zhou JY, Chong VF, Khoo JB. Indication of lower neck irradiation in nasopharyngeal carcinoma without nodal metastasis: the potential impact of tumor volume. Chin Med J (Engl). 2013;126(11):2120-2124.

13. Ramanah R, Berger MB, Chen L, Riethmuller D, Delancey JO. See it in 3D!: researchers examined structural links between the cardinal and uterosacral ligaments. Am J Obstet Gynecol. 2012;207(5):437 e431-437.

14. Day NJ, Earnshaw D, Salazar-Ferrer P, Walsh CJ. Preoperative mapping of fistula-in-ano: a new three-dimensional MRI-based modelling technique. Colorectal Dis. 2013;15(11):e699-701.

15. Hong G, Yang Z, Chu J, Li S, Zheng S, Zhou Z. Threedimensional MRI with contrast diagnosis of diseases involving peripheral oculomotor nerve. Clin Imaging. 2012;36(6):674-679.

16. Yilmaz KB, Canter HI, Vargel I, Ormeci T, Can U, Turk A, Saygili O. Use of three-dimensional MRI-angiography in preoperative evaluation and postoperative management of hemangiomas of head and neck region. J Craniofac Surg. 2011;22(5):1814-1818.

17. Giusti S, Forasassi F, Bastiani L, Cela V, Pluchino N, Fer- rari V, Fruzzetti E, et al. Anatomical localization of deep infiltrating endometriosis: 3D MRI reconstructions. Abdom Imaging. 2012;37(6):1110-1121.

18. Chen JH, Chen WP, Chan S, Yeh DC, Su MY, McLaren CE. Correlation of endogenous hormonal levels, fibroglandular tissue volume and percent density measured using 3D MRI during one menstrual cycle. Ann Oncol. 2013;24(9):2329-2335.

19. Clarke GM, Peressotti C, Mawdsley GE, Yaffe MJ. Design and characterization of a digital image acquisition system for whole-specimen breast histopathology. Phys Med Biol. 2006;51(20):5089-5103.

20. Clarke GM, Eidt S, Sun L, Mawdsley G, Zubovits JT, Yaffe MJ. Whole-specimen histopathology: a method to produce whole-mount breast serial sections for 3-D digital histopathology imaging. Histopathology. 2007;50(2):232-242.

21. Dahele M, Hwang D, Peressotti C, Sun L, Kusano M, Okhai S, Darling G, et al. Developing a methodology for three-dimensional correlation of PET-CT images and whole-mount histopathology in non-small-cell lung cancer. Curr Oncol. 2008;15(5):62-69.

22. Zhang Y, Hu J, Li J, Wang N, Li W, Zhou Y, Liu J, et al. Comparison of imaging-based gross tumor volume and pathological volume determined by whole-mount serial sections in primary cervical cancer. Onco Targets Ther. 2013;6:917-923.

23. Wang MY, Cheng JL, Han YH, Li YL, Dai JP, Shi DP. Measurement of tumor size in adult glioblastoma: classical cross-sectional criteria on 2D MRI or volumetric criteria on high resolution 3D MRI? Eur J Radiol. 2012;81(9):2370-2374. 